\title{
Die Petition zum Europäischen Parlament und die Justiziabilität von Entscheidungen des Petitionsausschusses
}

\author{
Oliver Mader
}

Für die Prüfung von Petitionen ist beim Europäischen Parlament der Petitionsausschuss zuständig. Erstmals sah die Geschäftsordnung der Gemeinsamen Versammlung der Europäischen Gemeinschaft für Kohle und Stahl im Jahr 1953 die Möglichkeit vor, Petitionen einzureichen. Seitdem hat das Europäische Parlament einige zehntausend Petitionen bearbeitet. Allein im Jahr 2010 wurden 1.655 und 2011 1.414 Petitionen neu eingereicht. Im Primärrecht ist die Petition mit Inkrafttreten des Vertrags von Maastricht vor 20 Jahren angekommen.

Das EP - weltweit die einzige direkt gewählte supranationale Institution - hat in den sechs Jahrzehnten seines Bestehens sowohl in seiner Rolle unter den Europäischen Organen als auch auf der internationalen Bühne kontinuierlich und erheblich an Bedeutung gewonnen. Es verlangt innerhalb der EU und geographisch weit über die EU hinausgehend die Beachtung von Grund- und Menschenrechten, ist aber auch selbst Grundrechtsverpflichteter, etwa in seiner Funktion als Adressat des Petitionsrechts.

Gemäß Art. 24 Abs. 2 AEUV besitzt jeder Unionsbürger „das Petitionsrecht beim Europäischen Parlament nach Art. 227 AEUV“. Danach kann jeder Bürger der Union sowie jede natürliche oder juristische Person mit Wohnort oder satzungsmäßigem Sitz in einem Mitgliedstaat „allein oder zusammen mit anderen Bürgern oder Personen in Angelegenheiten, die in die Tätigkeitsbereiche der Union fallen und die ihn oder sie unmittelbar betreffen, eine Petition an das Europäische Parlament richten“. Das Gemeinschaftsrecht eröffnete bereits seit 1953 durch Bestimmungen in der Geschäftsordnung der Gemeinsamen Versammlung der EGKS die Möglichkeit von Petitionen. ${ }^{1}$ Aufgrund der geringen Befugnisse der Versammlung konnte das Instrument allerdings in der Anfangszeit nur geringe Wirkung

1 Als historisches Konzept ist die Petition seit der Spätantike in der Form der Supplikation zum Kaiser (beziehungsweise seit 1521 zum Reichstag und dessen Supplikationsausschuss) bekannt, siehe Helmut Neuhaus, Reichstag und Supplikationsausschuß, Berlin 1977, S. 75 ff., S. 148 ff. Der Begriff „Petition“ als Forderung des Liberalismus gegenüber dem absolutistischen Staat wurde in Deutschland erst im 19. Jahrhundert - seinem genauen Inhalt nach allerdings als umkämpftes Recht - geläufig. Das Recht dem Parlament Beschwerden vorzubringen, wird in Titel $7 \$ 21$ der Bayerischen Verfassung von 1818 und in $\$ 159$ der Paulskirchenverfassung von 1849 genannt. Das Allgemeine Landrecht für die Preußischen Staaten vom 1. Juni 1794 stellte im Abschnitt „Verbrechen gegen die innere Ruhe und Sicherheit des Staats“ (zweiter Teil, Titel 20, \$ 156) fest, dass es jedem freistehe, seine „Zweifel, Einwendungen und Bedenklichkeiten“ sowie „Bemerkungen und Vorschläge über Mängel und Verbesserungen, sowohl dem Oberhaupte des Staats, als den Vorgesetzten der Departements anzuzeigen“. Auch wenn der Begriff der Petition hier nicht verwendet wird, ist bemerkenswert, dass sich anschließend im ALR eine statuierte Prüfpflicht findet: „letztere sind dergleichen Anzeigen mit erforderlicher Aufmerksamkeit zu prüfen verpflichtet“. Im englischen Recht ist der Begriff der Petition bereits seit 1272 als an das Parlament oder die Krone gerichtete Bittschrift nachweisbar, Paul Brand, Understanding Early Petitions: An Analysis of the Content of Petitions to Parliament in the Reign of Edward I, in: W. Mark Ormrod / Gwilym Dodd I Anthony Musson (Hrsg.), Medieval Petitions: Grace and Grievance, Woodbridge 2009, S. 99 119. 
entfalten. Ab 1989 legte eine interinstitutionelle Vereinbarung ${ }^{2}$ die formale Verpflichtung von Rat und Kommission fest, an der Behandlung von Petitionen aktiv mitzuwirken. Mit dem Vertrag von Maastricht (in Kraft getreten am 1. November 1993) fand das Petitionsrecht dann seine primärrechtliche ${ }^{3}$ Verankerung. Die Petition steht ihrer Natur nach in Nachbarschaft zu drei anderen in Art. 24 AEUV aufgelisteten Rechten: der mit dem Vertrag von Lissabon eingeführten Möglichkeit, eine Bürgerinitiative ${ }^{4}$ an die Kommission zu richten; dem Individualrecht, eine Beschwerde beim Europäischen Bürgerbeauftragten ${ }^{5}$ einzulegen; und dem Recht, sich in einer Vertragssprache ${ }^{6}$ an die Organe und Einrichtungen der Union zu wenden und die Antwort in derselben Sprache zu erhalten.

Die Handlungsoptionen, die dem Parlament bei der inhaltlichen Prüfung einer Petition offen stehen, sind nach allgemeiner Auffassung nicht rechtlicher, sondern politischer Natur. Es stellt sich folglich die Frage, inwieweit überhaupt Raum bleibt für eine rechtliche Nachprüfung der Entscheidungen über die beim EP eingebrachten Petitionen. Dies gilt insbesondere angesichts der Praxis des Petitionsausschusses, zulässige Petitionen ohne Prüfung unbehandelt zu lassen oder sie nach dem im Jahr 2011 eingeführten „Filterverfahren“ nach freiem Ermessen abzulegen beziehungsweise weiter zu verweisen. Als Antwort auf die jüngere Rechtsprechung zur Überprüfbarkeit von Handlungen des Petitionsausschusses des Parlaments, vor allem im Urteil zur Rechtssache Tegebauer ${ }^{7}$ und in der Rechtssache Schönberger $^{8}$, soll versucht werden, den gegenwärtigen Inhalt des Petitionsgrundrechts neu zu bestimmen und die Frage zu klären, welche Klagemöglichkeiten auf Nichtigerklärung von Entscheidungen des Petitionsausschusses offenstehen. Dabei ist eine Abgrenzung der Reichweite des Petitionsrechts im Hinblick auf das politische Ermessen des Petitionsausschusses zu unternehmen.

\section{Petitionsrecht und Petitionsverfahren}

\subsection{Grundrechtsnatur und Funktionen des Petitionsrechts als Unionsbürgerrecht}

Unionsbürgerrecht und Individualanspruch

Das Petitionsrecht steht systematisch in enger Beziehung zur Unionsbürgerschaft; so benennt Art. 24 Abs. 2 AEUV als Rechtsträger den „Unionsbürger“. Das Recht auf Petition kann insofern als eine Ausprägung der Unionsbürgerschaft mit Individualrechtsschutz verstanden werden. ${ }^{9}$ Nichts Anderes ergibt sich aus der anderen primärrechtlichen Grundlage

3 Art. 138d EGV alte Fassung, dessen Formulierung über Art. 194 EGV bis heute in Art. 227 AEUV beibehalten worden ist.

4 Art. 24 Abs. 1 AEUV in Verbindung mit Art. 11 Abs. 4 EUV.

5 Art. 24 Abs. 3 in Verbindung mit Art. 228 AEUV.

6 Art. 24 Abs. 4 AEUV, Vertragssprache: Art. 55 Abs. 1 EUV. Diese Rechte zählt (außer der Bürgerinitiative) auch Art. 20 Abs. 2 lit. d AEUV im Zusammenhang mit der Unionsbürgerschaft auf.

7 EuG, Rs. T-308/07, Tegebauer/Parlament vom 14. September 2011.

8 EuG, Rs. T-186/11, Schönberger/Parlament, Entscheidung zu erwarten im ersten Halbjahr 2013.

9 Anderer Auffassung offenbar Charlotte Gaitanides, in: Sebastian Heselhaus / Carsten Nowak, Handbuch der Europäischen Grundrechte, München 2006, S. 1318, die lediglich eine Verweisfunktion des Art. 24 EUV ohne spezifische Prägung der Unionsbürgerschaft annimmt; ebenso Armin Hatje, 
des Petitionsrechts, Art. 44 Grundrechtecharta. Das Institut der Unionsbürgerschaft selbst hat in jüngerer Zeit sowohl eine legitimatorische als auch eine inhaltliche Aufwertung erfahren, zu der die richterliche Rechtsfortbildung vom „Kernbestand“ der Unionsbürgerschaftsrechte zählt. Die Rechtsprechung nennt den Unionsbürgerstatus als „dazu bestimmt, der grundlegende Status der Angehörigen der Mitgliedstaaten zu sein “10. Trotz seiner subjektiv-rechtlichen Natur ist das Petitionsrecht dagegen nicht als ein Recht auf einen wirksamen Rechtsbehelf im Sinne des Art. 47 Abs. 1 Grundrechtecharta zu verstehen, weil das Petitionsrecht kein formales Rechtsmittel (vor Gericht) darstellt. ${ }^{11}$

Der Individualrechtscharakter impliziert, dass eine Petition nicht eine bloße allgemeine Anregung an das Parlament richtet, sich mit einem Thema zu befassen. Es begründet vielmehr als ein status activus politicus und subjektiv-öffentliches Bürgerrecht ${ }^{12}$ ein echtes Grundrecht ${ }^{13}$ mit der Funktion, an der politischen Willensbildung teilzunehmen. Gerade wegen dieser Partizipationsfunktion kann in ihm ein spezielles Element des Demokratieprinzips $^{14}$ gesehen werden. Diesem Umstand entspricht es, wenn die nach Art. 52 Abs. 2 Charta gegenüber dem Art. 44 Grundrechtecharta bedeutsamere Regelung des Art. 227 AEUV eine unmittelbare Betroffenheit des Petenten voraussetzt, die beinhaltet, dass die Angelegenheit auch für den Petenten selbst von Bedeutung ist. Institutionell trägt die dem Bürger eingeräumte Möglichkeit, sich mit seinem Petitum an das Parlament zu wenden, zur demokratischen Legitimation ${ }^{15}$ des Organs bei. In seiner Rechtsschutzfunktion zählt das Peti-

in: Jürgen Schwarze, EU-Kommentar, Baden-Baden 2012, Art. 24 EUV, Rn. 1. Offen bei Thomas Giegerich, in: Reiner Schulze / Manfred Zuleeg / Stefan Kadelbach, Europarecht Handbuch für die deutsche Rechtspraxis, Baden-Baden 2010, S. 372, Rn. 113. Es ist zwar zutreffend, dass das Petitionsrecht nicht streng an die Unionsbürgerschaft gebunden ist, sondern auch den Inhabern eines Wohnortes oder satzungsmäßigen Sitzes innerhalb der EU eingeräumt wird, Art. 201 Nr. 1 Geschäftsordnung des Parlaments (GeschOEP) vom Juli 2009. Auf die Qualität als individualschützendes Recht hat dieser Umstand jedoch keine Auswirkungen.

10 EuGH, Rs. C-184/99, Rudy Grzelczyk vom 20. September 2011, Rn. 31. Zur Zusammenführung von Unionsbürgerschaft und Grundrechtsschutz als Basis für einen grundrechtlichen Wesengehaltsschutz: Armin von Bogdandy / Matthias Kottmann / Carlino Antpöhler / Johanna Dickschen / Simon Hentrei / Maja Smrkolj, Ein Rettungsschirm für europäische Grundrechte - Grundlagen einer unionsrechtlichen Solange-Doktrin gegenüber Mitgliedstaaten, in: ZaöRV, 72. Jg. (2012), H. 1, S. $45-78$, S. $58 \mathrm{ff}$.

11 Hans Dieter Jarass, GRCh Kommentar, München 2010, Art. 47, Rn. 1, 5.

12 Vgl. Markus Kotzur, in: Rudolf Geiger / Daniel-Erasmus Khan / ders., EUV/AEUV, München 2010, Art. 227, Rn. 1; Peter Michael Huber, in: Rudolf Streinz, EUV/AEUV, München 2012, Art. 227 AEUV, Rn. 6; Axel Rolf Schneider, Petitionen zum Europäischen Parlament mit Berücksichtigung des Bürgerbeauftragten, Frankfurt am Main 2009, S. 108.

13 Vgl. Hans Dieter Jarass, a.a.O. (Fn. 11), Art. 44, Rn. 2. Charlotte Gaitanides geht von "grundrechtsgleicher Qualität" des Petitionsrechts aus (a.a.O., Rn. 2). Zur früheren Diskussion einer Individualrechtsfunktion des europäischen Petitionsrechts in der französischen Literatur: Hélène Surrel, in: RMC (revue du marché commun) 1990, S. 219 - 234, worin auch die rapide Entwicklung des Petitionsrechts hinsichtlich Verfahren, Prüfungsmaßstab und Rechtsförmigkeit in den letzten Jahren zum Ausdruck kommt.

14 Vgl. Peter Häberle, Europäische Verfassungslehre, Baden-Baden 2009, S. 136, im Zusammenhang mit legislativen Einzelmaßnahmen zur Behebung des Demokratiedefizits. Axel Rolf Schneider, a.a.O. (Fn. 12), S. 58 ff., betont außerdem die Integrationsfunktion des Petitionsrechts.

15 Wegen der Möglichkeit, das Handeln der Kommission zu überprüfen, wird von einem „Vehikel für die parlamentarische Kontrollfunktion des EP“" gesprochen: Peter Michael Huber, a.a.O. (Fn. 12), Rn. 5: „Demokratie-, Partizipations- oder Legitimationsfunktion“. 
tionsrecht außerdem zu den Mechanismen des Rechtsstaatsprinzips ${ }^{16}$, das es dem Unionsbürger außergerichtlich und auf einer niedrigen Eingangsschwelle ermöglicht, seine Rechte zu verfolgen beziehungsweise Entwicklungen anzustoßen.

\section{Institutioneller Rahmen und Rechtsstaatsfunktionen}

Für die Wahrnehmung des Petitionsrechts ist keine anwaltliche Vertretung erforderlich, sind keine Gebühren zu entrichten und keine speziellen Formen ${ }^{17}$ oder Fristen zu beachten. Das institutionelle Gleichgewicht (Gewaltenteilung) und der Schutz der richterlichen Unabhängigkeit erfordern lediglich eine systematische Beschränkung ${ }^{18}$ des Petitionsrechts dahingehend, dass es nicht parallel zu einem anhängig gemachten Gerichtsverfahren betrieben werden darf. ${ }^{19}$

Der nach dem internen Organisationsrecht für Petitionen zuständige Petitionsausschuss ${ }^{20}$ würde bei gerichtlicher Anhängigkeit einer Sache die Behandlung der Petition aussetzen müssen, bis das Gerichtsverfahren abgeschlossen ist. Normativ-institutionell ist die Einrichtung des Petitionsausschusses des EP im Gegensatz zum Petitionsausschuss des Deutschen Bundestages (Art. 45c Abs. 1 GG) nicht auf konstitutioneller Ebene, sondern nur in der Geschäftsordnung des Parlaments (dort in Art. 183 und Art. 201 bis 203) verankert. Der Petent hält nach Durchlaufen des Petitionsverfahrens allenfalls eine unverbindliche Entscheidung des Petitionsausschusses zu seinen Gunsten in Händen. Diese kann aber die exekutiv entscheidenden Stellen zu einer Änderung ihrer Haltungen (und entsprechenden Entscheidungen) bewegen. Das mit einem gerichtlichen Verfahren verbundene direkte Kostenrisiko

16 Nach Art. 2 EUV sind Demokratie und Rechtsstaatsprinzip Werte, auf die sich die Union gründet. Diese den Individualrechten zugrundeliegenden Werte des Art. 2 EUV (also etwa das Rechtsstaatsprinzip) sind ähnlich wie die Ziele der Union (Art. 3 EUV) nicht als solche justiziabel. Vgl. Matthias Ruffert, in: Christian Calliess / Matthias Ruffert, Verfassung der Europäischen Union, München 2006, Art. I-3, Rn. 4 f.

17 Zum strittigen Schriftformerfordernis bei der Einlegung einer Petition: Annette Guckelberger, Europäischer Bürgerbeauftragter und Petitionen, Berlin 2004, S. 62. Für die Möglichkeit einer auch mündlichen Einlegung plädieren Peter Michael Huber, a.a.O. (Fn. 12), Rn. 16; Axel RolfSchneider, a.a.O. (Fn. 12), S. $135 \mathrm{f}$.

18 Im Gegensatz zur Regelung des Verfahrens vor dem Bürgerbeauftragten ist die genannte Einschränkung der Zuständigkeit (Unzulässigkeit bei anhängigen Gerichtsverfahren) weder im Primärrecht noch in der GeschOEP zur Petition enthalten. Wie hier: Siegfried Magiera, in: Hans Meyer (Hrsg.), Charta der Grundrechte der Europäischen Union, Baden-Baden 2011, Art. 44, Rn. 10 mit weiteren Nachweisen; Walter Frenz, Handbuch Europarecht, Berlin / Heidelberg 2009, S. 1449 , Rn. 4785.

19 Insofern und in einigen anderen Punkten gibt es Parallelen zum Beschwerdeverfahren vor dem europäischen Bürgerbeauftragten, vgl. Art. 228 Abs. 1 UA 2 AEUV und Art. 1 Abs. 3 des Beschlusses des Parlaments über die Regelungen und allgemeinen Bedingungen für die Ausübung der Aufgaben des Bürgerbeauftragten vom 9. März 1994 (ABl. Nr. L 113 vom 4. Mai 1994, S. 15), in der Fassung des Beschlusses vom 18. Juni 2008 (ABl. Nr. L 189 vom 17. Juli 2008, S. 25). Ein wesentlicher Unterschied der Petition gegenüber der Beschwerdeerhebung zum Bürgerbeauftragten besteht darin, dass dieser nur Missstände in der Verwaltungstätigkeit der EUEinrichtungen untersucht.

20 Art. 202 Nr. 1 GeschOEP. Gemäß Art. 183 und Annex VII Ziffer XX der GeschOEP ist er als ständiger Ausschuss zuständig sowohl für Petitionen als auch für die Beziehungen zum Europäischen Bürgerbeauftragten. Gegenwärtig besteht er aus 35 Mitgliedern und 29 Stellvertretern, er wird von der Italienerin Erminia Mazzoni geleitet. 
wird vermieden, und die Petition steht auch dann noch zur Verfügung, wenn beispielsweise Fristen für die Klageerhebung verstrichen oder andere Voraussetzungen für eine Klage nicht gegeben sind. Ferner erhält das Petitionsrecht dort seine eigenständige Bedeutung, wo es nicht um rechtliche Zusammenhänge geht, sowie dort, wo Ermessensentscheidungen in Frage gestellt werden sollen. ${ }^{21}$ Das Petitionsrecht ist insofern geeignet, Rechtsschutzlücken zu schließen. ${ }^{22}$ Über den individuellen Rechtsschutz hinaus erfüllt es Funktionen der parlamentarischen Kontrolle ${ }^{23}$ und der demokratischen Rückkopplung. ${ }^{24}$ Das Plenum wird bei der Entscheidung über eine Petition in der Regel ${ }^{25}$ nicht beteiligt, der Ausschuss erstattet dem Parlament halbjährlich über die Ergebnisse seiner Beratungen Bericht. ${ }^{26}$ Inhaltlich liefern die Bereiche Grundrechte/Justiz, Binnenmarkt und Umweltschutz über die Jahre hinweg wichtige Themenschwerpunkte. ${ }^{27}$ Quantitativ werden die meisten Petitionen von deutschen, spanischen und italienischen Unionsbürgern eingereicht, die zusammengenommen etwa 50 Prozent der Petitionen stellen.

\section{Keine Kassationsbefugnis}

Das EP beziehungsweise sein Petitionsausschuss hat keinerlei Befugnis zur Kassation einer mit der Petition kritisierten Verwaltungsentscheidung. Allerdings steht ihm im Rahmen des parlamentarischen Selbstbestimmungsrechts eine Fülle von Reaktionsmöglichkeiten dafür zur Verfügung, wie es Untersuchungen anstellen und mit politischen Argumenten zu einer Petition Stellung beziehen kann, wie es auf die Beseitigung eines Missstands Einfluss nehmen oder eine Anregung zu legislativem Tätigwerden aufnehmen kann. Es kann dazu die Kommission ${ }^{28}$ um Unterstützung bitten, was dazu führen kann, dass diese ein Vertragsverletzungsverfahren gegen einen oder mehrere Mitgliedstaaten einleitet. Der Petitionsausschuss kann über den Parlamentspräsidenten seine Stellungnahmen oder Empfehlungen der Kommission, dem Rat, einem anderen Ausschuss oder einer nationalen Behörde übermitteln, „um ein Tätigwerden oder eine Antwort zu erwirken“29.

$21 \mathrm{Zu}$ diesen und weiteren strategischen Abwägungen bei der Wahrnehmung des Petitionsrechtes: Axel Rolf Schneider, a.a.O. (Fn. 12), S. 56 ff.

22 Vgl. Walter Frenz, a.a.O. (Fn. 18), Bd. 6, S. 80, Rn. 291.

23 Vgl. Hans Dieter Jarass, a.a.O. (Fn. 11), Art. 44, Rn. 2; Walter Frenz, a.a.O. (Fn. 18), Bd. 4, S. 1443, Rn. 4762.

$24 \mathrm{Zu}$ Begriff und Funktionen der Petition: Charlotte Gaitanides, a.a.O. (Fn. 9), S. 1319 f. Saskia Eckhardt, Die Akteure des außergerichtlichen Grundrechtsschutzes in der Europäischen Union, Frankfurt am Main 2010, S. $288 \mathrm{ff}$.

25 Der Ausschuss kann allerdings einen Initiativbericht ausarbeiten und dem Plenum gemäß Art. 202 Nr. 2 GeschOEP einen „kurzen Entschließungsantrag“ vorlegen. Wird ein Bericht vorgelegt so kann der assoziierte Ausschuss unter bestimmten Umständen seine Vorschläge unmittelbar im Plenum einreichen, Art. 202 Nr. 3 GeschOEP.

26 Art. 202 Nr. 8 GeschOEP.

27 Berichtsentwurf für das Jahr 2011, 2011/2317(INI) vom 29. März 2012, S. 8. Aus dem Bericht für 2010, 2010/2295(INI) vom 17. Juni 2011 und dem Berichtsentwurf für das Jahr 2011 geht hervor, dass im Jahr 20101.655 Petitionen eingereicht wurden, von denen 40 Prozent unzulässig waren; von den im Jahr 2011 eingereichten 1.414 Petitionen waren 29 Prozent unzulässig.

28 Art. 202 Nr. 6 GeschOEP.

29 Art. 202 Nr. 7 GeschOEP. 


\subsection{Verweisungsmöglichkeiten und neu eingeführtes „Filterverfahren“}

Die an ein EU-Organ oder eine nationale Behörde gerichtete Bitte um Tätigwerden kann Teil einer Behandlung der Petition darstellen und ist nicht mit einer Verweisung zu verwechseln. Will der Petitionsausschuss eine Sache lediglich weiterverweisen, so sind ihm Grenzen gesetzt: Der Ausschuss kann die Angelegenheit nach Zweckmäßigkeitserwägungen lediglich an den Bürgerbeauftragten ${ }^{30}$ (nur an ihn) weiterleiten, wenn er der Auffassung ist, dass dieser dem Anliegen des Petenten besser entsprechen kann. Der Ausschuss kann in seiner Stellungnahme seine Meinung dazu festhalten, welche andere (auch nationale) Stelle für eine Behandlung beziehungsweise Weiterbehandlung besser geeignet sei. Jedoch ist die Option der bloßen Abgabe an andere Stellen als eine Ausnahme von der eigenen Behandlung durch den Petitionsausschuss eng zu fassen. Für die Entscheidung, eine Angelegenheit an andere Stellen als den Bürgerbeauftragten weiterzuleiten, ist angesichts des Wortlauts von Art. 201 Nr. 12 der Geschäftsordnung kein Raum; sie würde den Petenten, ohne dass er eine Bescheidung der strukturell dafür vorgesehenen beiden Einrichtungen (Petitionsausschuss und Bürgerbeauftragter) erhielte, lediglich in eine Verweisung senden, die er selbst nicht gewählt hat. ${ }^{31}$ Bei Verweisung an den Bürgerbeauftragten verbleibt es dagegen bei einer dem EP zugeordneten Einrichtung, die ihm berichtet. ${ }^{32}$ Verfahrenstechnisch ist für die Abgabe an den Bürgerbeauftragten das Einverständnis des Petenten einzuholen, eine automatische Weiterleitung seiner Petition an ihn im Sinne „kommunizierender Röhren“ ist abzulehnen. ${ }^{33}$

Im Juni 2011 führte das EP ein neues „Filterverfahren“34 ein mit dem Ziel, die Arbeitsbelastung des Ausschusses zu senken, indem „Eingaben, die als nicht relevant erachtet werden, zur Beantwortung an andere Dienste " 35 weitergeleitet werden können. In diesem $\mathrm{Zu}$ sammenhang wurden im Jahr 2011 insgesamt 647 aussortiert und nicht als Petitionen registriert. Bei 57 davon handelte es sich um Weiterleitungen an den Bürgerbeauftragten. Die eingangs erwähnte Zahl von 1.414 Petitionen im Jahr 2011 umfasst nur die offiziell als Petition registrierten Eingaben. ${ }^{36}$ Gerade angesichts dieses neuen Filterverfahrens und den mit ihm verbundenen Gefahren für die umfassende Gewährleistung des Petitionsrechts be-

30 Art. 201 Nr. 12 GeschOEP.

31 Anderer Auffassung offenbar Charlotte Gaitanides, a.a.O. (Fn. 9), S. 1328, Rn. 36, die auch eine Übermittlung ,an nationale Parlamente oder Einrichtungen “ für zulässig hält, während diese nach hier vertretener Auffassung nur bei inhaltlicher Stellungnahme durch den Ausschuss möglich ist. Ähnlich wie hier Walter Frenz, a.a.O. (Fn. 18), Bd. 5, S. 804, Rn. 2770.

32 Vgl. Art. 3 Nr. 7, 8 und 4 des Beschlusses des Europäischen Parlaments über die Regelungen und allgemeinen Bedingungen für die Ausübung der Aufgaben des Bürgerbeauftragten vom 9. März 1994, ABl. Nr. L 113 vom 4. Mai 1994, S. 15, zuletzt geändert durch Beschluss vom 18. Juni 2008, ABl. Nr. L 189 vom 17. Juli 2008, S. 25.

33 Wie hier Walter Frenz, a.a.O. (Fn. 18), Bd. 5, S. 804, Rn. 2770; Annette Guckelberger, Das Petitionsrecht zum Europäischen Parlament sowie das Recht zur Anrufung des Europäischen Bürgerbeauftragten im Europa der Bürgerin, in: DÖV 2003, S. 829 - 838, S. 837 f.; Axel Rolf Schneider, a.a.O. (Fn. 12), S. 215.

34 Berichtsentwurf des Petitionsausschusses für das Jahr 2011, 2011/2317(INI) vom 29. März 2012, S. $16 \mathrm{ff}$.

35 Ebenda, S. $16 \mathrm{f}$.

36 Zum Vergleich: der Bürgerbeauftragte erhielt 2.510 Beschwerden im Jahr 2011 und 2.667 im Jahr 2010. Vgl. Jahresbericht des Bürgerbeauftragten 2011 vom 13. Februar 2012, S. 22; Jahresbericht 2011 vom 14. Februar 2011, S. 19. 
steht umso größerer Bedarf an einer trennscharfen Abgrenzung des Grundrechts, unter Berücksichtigung der hier dargelegten Inhalte des Rechts und der aus rechtlichen Gründen beschränkten Verweisungsmöglichkeiten einer Petition an andere Einrichtungen. Die klare Abgrenzung zur „Nicht-Petition“ trägt dazu bei, die Arbeitsfähigkeit des Ausschusses und damit die effektive Behandlung von (echten) Petitionen zu befördern. Die oben genannte Möglichkeit der Einschätzung einer Eingabe als „nicht relevant“ wird in diesem Zusammenhang allerdings nicht als maßgebliches Differenzierungskriterium standhalten können. Es ist zwar nicht zu beanstanden, wenn Petitionen zu ähnlichen Themen zusammengefasst werden, um sie in ähnlicher Weise zu behandeln. Dies setzt aber eben die tatsächliche Ähnlichkeit der Petitionen voraus und darf nicht im Ergebnis zu einer Beliebigkeit bei der thematischen Einordnung oder zu einer willkürlichen Ablage beziehungsweise Verweisung der einzelnen Petition führen.

\subsection{Zulässiger Petitionsgegenstand und Petitionsinteresse}

Als zulässige Gegenstände einer Petition nennt das Primärrecht in Art. 227 AEUV solche „Angelegenheiten, die in die Tätigkeitsbereiche der Union fallen“ und die den Petenten „unmittelbar betreffen“. Das EP selbst fasst beide Voraussetzungen weit auf ${ }^{37}$ und bezieht etwa auch die Umsetzung und den Vollzug von EU-Akten durch die Mitgliedstaaten mit in die Tätigkeitsbereiche der Union ein. Der Juristische Dienst des Parlaments zieht für die Frage des Zuständigkeitsbereichs bei Petitionen die Ziele der Union nach Art. 3 EUV in Verbindung mit Art. 2 bis Art. 6 AEUV heran und konstatiert, wohl mit ähnlichem Ergebnis: „Die Summe der Bestimmungen, die in diesen Artikeln des Vertrags enthalten ist, schafft einen Tätigkeitsbereich, der über die Kompetenzbereiche der Union hinausgeht. “38

Die Voraussetzung der unmittelbaren Betroffenheit als Petitionsbefugnis liegt bereits dann vor, wenn die Person eine ernstzunehmende und tatsächliche Besorgnis in Bezug auf das Begehren darlegen kann. ${ }^{39}$ Ein bloßes Auskunftsersuchen oder eine Kommentierung von Umständen oder Politiken fällt nicht unter das Petitionsrecht. ${ }^{40}$ Aber eine Petition braucht auch nicht formal als solche benannt zu werden. Insoweit Art. 44 Grundrechtecharta von den genannten Zulässigkeitsvoraussetzungen absieht, ist Art. 52 Abs. 2 Grundrechtecharta maßgeblich, der die Ausübung der in der Charta vorgesehenen Rechte in den

37 Vgl. Thomas Giegerich, a.a.O. (Fn. 9), S. 373, Rn. 117; Peter Michael Huber, a.a.O. (Fn. 12), Art 227 AEUV, Rn. 11, 14. Historisch gesehen ist das Parlament selbst mit der Erweiterung des sachlichen Anwendungsbereichs vorangegangen, indem es in seiner internen Geschäftsordnung (damals Art. 191 Abs. 1 GeschOEP alt) über die damals geltende enge Fassung des Art. 194 EG („die in den Tätigkeitsbereiche der Gemeinschaft fallen“) hinaus bereits alle Tätigkeitsbereiche der Union in den Anwendungsbereich aufgenommen hatte.

38 Zitiert nach dem Berichtsentwurf des Petitionsausschusses für das Jahr 2011, 2011/2317(INI) vom 29. März 2012, S. 18.

39 Vgl. Thomas Giegerich, a.a.O. (Fn. 9), S. 373, Rn. 117. Nach Charlotte Gaitanides, a.a.O. (Fn. 9), S. 1324, Rn. 22 genügt es, wenn die Angelegenheit „für den Petenten in objektiver und nachvollziehbarer Weise von nicht ganz unerheblicher Bedeutung ist“. Für das Verfahren vor dem Bürgerbeauftragten ist eine unmittelbare Betroffenheit keine Beschwerdevoraussetzung, Johann Schoo, in: Jürgen Schwarze (Hrsg.), a.a.O. (Fn. 9), Art. 228 AEUV, Rn. 16.

40 Vgl. Axel Rolf Schneider, a.a.O. (Fn. 12), S. 67. 
Rahmen der in den Verträgen festgelegten Bedingungen und Grenzen stellt. ${ }^{41}$ Dadurch wird also lediglich bestätigt, dass die in den Verträgen genannten Zulässigkeitsvoraussetzungen für die Einlegung einer Petition zu beachten sind. Allerdings ist daran zu denken, dass sich das EP unter Umständen durch eine großzügigere Handhabung seiner Verwaltungspraxis bezüglich bestimmter Zulässigkeitsvoraussetzungen in dieser Richtung selbst bindet.

Darüber hinaus enthält die Geschäftsordnung des Parlaments, der historisch die Statuierung des Petitionsrechts als Ausfluss des parlamentarischen Selbstorganisationsrechts entstammt, weitere Voraussetzungen: Petitionen müssen die Namen, die Staatsangehörigkeit und den Wohnsitz aller Petenten enthalten und grundsätzlich in einer Amtssprache der Europäischen Union abgefasst sein. ${ }^{42}$ Die zulässigen Petitionen werden in der Reihenfolge ihres Eingangs in ein Register eingetragen; unzulässige Petitionen legt der Ausschuss ab und teilt dies den Petenten mit Begründung mit. ${ }^{43}$

Der Ausschuss hat sich an den Gegenstand der Petition zu halten, er darf nicht aus eigener Initiative (ohne eingelegte Petition) Untersuchungen einleiten oder Ermittlungen über den Gegenstand einer Petition hinaus anstellen. Es steht ihm allerdings frei, sich auch mit unzulässigen Petitionen zu befassen (zum Beispiel von Personen, denen territorial kein Petitionsrecht zusteht). Dem Petenten erwächst in solchen Fällen aber weder ein Recht auf Prüfung, noch sind andere Organe, also etwa Rat und Kommission, verpflichtet, daran mitzuwirken. Der Grund dafür ist, dass das Selbstorganisationsrecht des Parlaments nicht

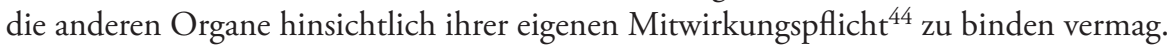

Ein Sonderfall der Genese einer Petition und ihres Gegenstandes ergibt sich aus der Nähe zum direkt-demokratischen Institut der Bürgerinitiative. Eine EU-Bürgerinitiative (Art. 24 Abs. 1 AEUV i.V.m. Art. 11 Abs. 4 EUV), die nicht die Voraussetzungen des Art. 9 der Verordnung über die Bürgerinitiative ${ }^{45}$ erfüllt, weil nicht alle in der Verordnung genannten Verfahren und Bedingungen eingehalten worden sind, wird der Kommission nicht zur Erarbeitung eines Legislativvorhabens vorgelegt. Ein solcher, originär einer Bürgerinitiative zuzurechnende Gegenstand kann laut Geschäftsordnung vom Petitionsausschuss geprüft werden, „wenn dieser eine Weiterbehandlung für angebracht erachtet“ ${ }^{\text {“6 }}$. Diejenigen Bürgerinitiativen, die die Voraussetzungen des Art. 9 der Verordnung über die Bürgerinitiative erfüllen und daher zu einer Aufforderung der Kommission führen, ein Legislativvorhaben zu erarbeiten, sind vom Petitionsausschuss daraufhin zu überprüfen, ob sie sich auf die Arbeiten des Ausschusses auswirken können. Petenten, die Petitionen zu verwandten Themen eingereicht haben, sind davon gegebenenfalls in Kenntnis zu setzen. ${ }^{47}$

41 Art. 227 AEUV ist insofern lex specialis zu Art. 44 Grundrechtecharta, Peter Michael Huber, a.a.O. (Fn. 12), Art 227 AEUV, Rn. 2.

42 Art. 201 Nr. 2, 5 GeschOEP. Gegen eine sekundärrechtliche Ausgestaltung und Erweiterung des grundrechtlichen Schutzes ist nichts einzuwenden, eine Verengung des Schutzbereichs auf diesem Wege wäre jedoch abzulehnen.

43 Art. 201 Nr. 6 GeschOEP. Zur gerichtlichen Überprüfbarkeit der Entscheidung über die Unzulässigkeit, siehe unter Abschnitt 2.2., Unterpunkt „Szenarien für die Behandlung von Petitionen“.

44 Art. 202 Nr. 6, 7 GeschOEP.

45 Verordnung (EU) Nr. 211/2011 des Europäischen Parlaments und des Rates vom 16. Februar 2011 über die Bürgerinitiative, ABl. Nr. L 65 vom 11. März 2011, S. 1 - 22.

46 So Art. 203a Abs. 2 GeschOEP.

47 Art. 203a Abs. 1 GeschOEP. 


\subsection{Schutzbereich des Petitionsrechts}

\section{Persönlicher Schutzbereich}

Unionsbürger haben das Petitionsrecht ebenso wie andere natürliche Personen, denen ihr Wohnsitz innerhalb der EU einen legitimierenden Anknüpfungspunkt für ihre Einbeziehung in den Schutzbereich bietet. Es kommt also lediglich auf eine tatsächliche Wohnung in mindestens einem Mitgliedstaat an; der Petent braucht keinen Wohnsitz im Rechtssinne nachzuweisen. ${ }^{48}$ In ähnlicher Weise sind auch juristische Personen als Grundrechtsberechtigte mit umfasst, deren satzungsmäßiger Sitz innerhalb der EU liegt. ${ }^{49}$ Der Begriff „satzungsmäßiger Sitz" wird weit gefasst. Eine Unterscheidung in juristische Personen des Privatrechts und des öffentlichen Rechts der Mitgliedstaaten findet nicht statt: Beide sind grundsätzlich petitionsberechtigt. Der Grund dafür liegt darin, dass juristische Personen des öffentlichen Rechts der Mitgliedstaaten institutionell keine Hoheitsträger der Union sind, selbst wenn sie im Einzelfall Unionsrecht ausführen. Die Hoheitsträger der Union selbst sind nicht petitionsberechtigt. Unter den nationalen juristischen Personen des öffentlichen Rechts sind die EU-Mitgliedstaaten von der Petition ausgeschlossen ${ }^{50}$, einerseits weil sie sowohl als „Herren der Verträge“ originär unionsverfassungsrechtliche Hoheitsgewalt innehaben, andererseits weil ihnen in den Verträgen ein laut Art. 344 AEUV abschließend gemeinter Katalog von Rechtsmitteln (darunter vor allem das Vertragsverletzungsverfahren nach Art. 263 Abs. 2 AEUV) zur Verfügung steht.

\section{Sachlicher Schutzbereich und Eingriffe}

Der sachliche Schutzbereich des Petitionsrechts umfasst nach allgemeiner Ansicht ${ }^{51}$ (1) die Entgegennahme der Petition, (2) wenn sie zulässig ist, ihre sachliche Prüfung und (3) eine inhaltliche Stellungnahme, die dem Petenten in der Sprache, die er zulässigerweise für seine Petition gewählt hat, mitzuteilen ist. Mit diesem Dreiklang ist der von Rechts wegen durchsetzbare Teil des Petitionsrechts benannt. Ein über Kenntnisnahme, Prüfung beziehungsweise Behandlung der Petition und Bescheidung hinaus gehender Anspruch, etwa auf Abhilfe

48 Wie hier Axel Rolf Schneider, a.a.O. (Fn. 12), S. 111 f.; das Parlament fordert dagegen über den Wortlaut des Wohnsitzerfordernisses hinaus, dass der Petent sich rechtmäßig in einem Mitgliedstaat aufhalten müsse, vgl. Entschließung des Europäischen Parlaments zu den Beratungen des Petitionsausschusses im parlamentarischen Jahr 1994-1995, vom 12. Juli 1995, ABl. Nr. C 249 vom 25. September 1995, S. 71.

49 Art. 201 Nr. 13 GeschOEP geht darüber hinaus von der Möglichkeit einer Behandlung von Petitionen Gebietsfremder aus, die „getrennt erfasst und getrennt abgelegt" werden. Für diese besteht kein Anspruch auf Prüfung, aber eine dem Parlament im Rahmen seiner Organisationsgewalt (Art. 14 Abs. 1 EUV) zustehende Befugnis sich mit der Sache dennoch zu befassen. Zur strittigen (historischen) Diskussion über die Petitionsberechtigung juristischer Personen: Sven Hölscheidt, Die Ausgestaltung des Petitionsrechts in der EU-Grundrechtecharta, in: EuR 2002, S. 440 - 448, S. 443 f.; Jon Meese, Das Petitionsrecht, Frankfurt am Main 2000, S. 75 ff.

50 Vgl. Annette Guckelberger, a.a.O. (Fn. 33), S. 832; Axel Rolf Schneider, a.a.O. (Fn. 12), S. 114.

51 Vgl. Art. 202 Nr. 1 und Nr. 9 GeschOEP. Thomas Giegerich, a.a.O. (Fn. 9), S. 374, Rn. 119; Charlotte Gaitanides, a.a.O. (Fn. 9), S. 1328, Rn. 35, 39 f.; Marcel Haag, in: Hans von der Groeben I Jürgen Schwarze, EUV/EGV, Baden-Baden 2004, Art. 194, Rn. 8. 
in der vorgebrachten Sache existiert nicht. ${ }^{52}$ Das gilt auch dann, wenn das Parlament beziehungsweise der Petitionsausschuss das Anliegen des Petenten an sich als berechtigt anerkennt. Allerdings beinhaltet die Abwehrfunktion des Petitionsrechts, dass auch der Vorgang der Einlegung der Petition und seine vorbereitenden Maßnahmen vom Schutzbereich mitumfasst sind. ${ }^{53}$ In diesem Sinne ist etwa bei zu befürchtender Viktimisierung des Petenten die Möglichkeit gegeben, die Petition vertraulich zu behandeln. Eingriffe in das Recht sind sowohl beim „Ob“ der Gewährung des Petitionsrechts als auch bei der Art der Behandlung des Petenten im laufenden Verfahren denkbar. Ausdrückliche Schranken des Petitionsrechts gibt es nicht. Anwendbar scheint aber eine allgemeine Schrankensystematik, wonach „zwingende Gründe des Allgemeinwohls“ eine Einschränkung des Petitionsrechts rechtfertigen könnten, ähnlich wie beim ungeschriebenen Rechtfertigungsgrund im Rahmen der Einschränkung von Grundfreiheiten. ${ }^{54}$

\subsection{Das Verfahren der Prüfung von Petitionen}

Das Primärrecht enthält keine Vorschriften zur Ausgestaltung des Petitionsverfahrens und überlässt die prozedurale Gestaltung damit dem Selbstorganisationsrecht des Parlaments in seiner Geschäftsordnung. ${ }^{55}$ Wesentliche Änderungen hat es im Verfahren in den letzten Jahren nicht gegeben. ${ }^{56}$ Der Petitionsausschuss prüft die für zulässig befundenen Petitionen entweder per Aussprache während einer ordentlichen Sitzung oder im Wege des schriftlichen Verfahrens. Dabei können die Petenten zu den betreffenden Ausschusssitzungen eingeladen werden oder eine solche Teilnahme beantragen. Sie können nach dem Ermessen des Ausschuss-Vorsitzes das Wort erhalten. ${ }^{57}$ Der Ausschuss kann sodann einen Initiativbericht ausarbeiten, dem Parlament einen Entschließungsantrag vorlegen oder die Stellungnahme eines anderen Ausschusses einholen ${ }^{58}$ beziehungsweise - wenn die Anwendung oder Auslegung des Unionsrechts oder Vorschläge zur Änderung des geltenden Rechts betroffen sind - den für den Gegenstand zuständigen Ausschuss assoziieren. ${ }^{59}$

Der Ausschuss kann zur Prüfung von Petitionen, zur Tatsachenfeststellung oder Ermittlung von Lösungen Informationsbesuche in dem betreffenden Mitgliedstaat oder der Re-

52 Vgl. Johann Schoo / Niklas Görlitz, in: Jürgen Schwarze (Hrsg.), a.a.O. (Fn. 9), Art. 227 AEUV, Rn. 14; Philipp Voet van Vormizeele, in: Jürgen Schwarze (Hrsg.), a.a.O. (Fn. 9), Art. 44 GrCh, Rn. 3; Jon Meese, a.a.O. (Fn. 49), S. 69.

53 Vgl. Axel Rolf Schneider, a.a.O. (Fn. 12), S. 103.

54 Vgl. Walter Frenz, a.a.O. (Fn. 18), Bd. 4, Berlin u.a. 2009, S. 1450, Rn. 4787.

55 Außerdem hat sich das Parlament eine interne Leitlinie (PE 380.652) zur praktischen Handhabung des Verfahrens gegeben, die hier aber aufgrund des unverbindlichen Charakters dieses Dokuments unberücksichtigt bleiben soll. Seine Regelungen haben in jedem Fall mit höherrangigem Recht in Einklang zu stehen.

56 Eine Reforminitiative des Parlaments zur verkürzten, transparenteren und effektiveren Gestaltung des Verfahrens liegt mit dem Ausschussdokument A5-0088/2001 vom 19. März 2001 vor, „über die Petition als Institution zu Beginn des 21. Jahrhunderts“. Bislang hatte diese aber - soweit ersichtlich - keine weiteren Folgen.

57 Art. 202 Nr. 1 GeschOEP. Andere Bürger können sich den in einem elektronischen Register eingetragenen Petitionen anschließen, Art. 202 Nr. 4 GeschOEP.

58 Art. 202 Nr. 2 GeschOEP, nach der hier vertretenen Ansicht stehen dem Ausschuss diese Optionen über den Wortlaut hinaus sowohl bei zulässigen als auch bei unzulässigen Petitionen offen.

59 Art. 202 Nr. 3 GeschOEP. 
gion durchführen. ${ }^{60}$ Er kann die Unterstützung der Kommission suchen, „insbesondere durch Klarstellungen zur Anwendung oder Einhaltung des Unionsrechts und durch Übermittlung sämtlicher Informationen und Unterlagen zum Gegenstand der Petition "61. Über den Parlamentspräsidenten kann der Ausschuss seine Stellungnahme oder Empfehlung der Kommission, dem Rat oder der betroffenen nationalen Behörde übermitteln lassen, um ein Tätigwerden oder eine Antwort zu erwirken. ${ }^{62}$ Die anderen Organe und die Behörden der Mitgliedstaaten sind im Sinne der Organtreue (Art. 13 Abs. 2 EUV) beziehungsweise der Unionstreue (Art. 4 Abs. 3 EUV) und nach dem Grundsatz der loyalen Zusammenarbeit ${ }^{63}$ zur Mitwirkung verpflichtet. Zum Abschluss des Verfahrens werden die Petenten über den vom Ausschuss gefassten Beschluss und dessen Begründung unterrichtet. ${ }^{64}$ Sodann wird die Petition, wiederum mit Benachrichtigung der Petenten, für abgeschlossen erklärt.

In das Register eingetragene Petitionen werden dem Parlament in Plenarsitzung bekannt gegeben. Das Parlament macht, mit dem Einverständnis der Petenten, Titel und Zusammenfassung des Inhalts von Petitionen, die Stellungnahmen und die wichtigsten Beschlüsse in einer Datenbank öffentlich zugänglich. Die vertraulich zu behandelnden Petitionen können nur die Abgeordneten im Archiv einsehen.

\section{Kontrolle von Petitionsentscheidungen}

Als Organe für die Überprüfung von Handlungen des Petitionsausschusses, insbesondere von Entscheidungen über Petitionen, kommen einerseits das Plenum des EP, andererseits der Europäische Gerichtshof in Frage: das Parlament deswegen, weil es für die Einsetzung seiner Ausschüsse zuständig ist (Art. 183 GeschOEP) und insofern eine institutionelle Verantwortung für den Ausschuss trägt; dem Gerichtshof obliegt es, die Wahrung des Unionsrechts zu sichern (Art. 19 EUV).

60 Art. 202 Nr. 5 GeschOEP. Im Sinne der Implied-Powers-Lehre stehen dem Organ diejenigen Befugnisse der Informationsbeschaffung zur Verfügung, die zur wirksamen Erfüllung ausdrücklich eingeräumter Aufgaben (hier Art. 227 AEUV) erforderlich sind, vgl. EuGH, Rs. 8/55, Fédération Charbonnière de Belgique vom 29. November 1956, S. 302, S. 312. Dies ist jedoch strittig, Walter Frenz lehnt eine Ableitung des Petitionsinformationsrechts des Parlaments aus der ImpliedPowers-Lehre als unvereinbar mit dem Grundsatz der begrenzten Einzelermächtigung ab: Walter Frenz, a.a.O. (Fn. 18), Bd. 4, S. 1445, Rn. 4770, Fußnote 750. Zur Klagemöglichkeit des Parlaments bei Kooperationsverweigerung durch andere Unionsorgane: Axel RolfSchneider, a.a.O. (Fn. 12), S. $148 \mathrm{ff}$.

61 Art. 202 Nr. 6 GeschOEP.

62 Art. 202 Nr. 7 GeschOEP.

63 EuGH, Rs. 230/81, Luxemburg/Parlament vom 10. Februar 1983, Rn. 37; Siegfried Magiera, a.a.O. (Fn. 18), Art. 44, Rn. 14.

64 Art. 202 Nr. 9 GeschOEP; zur Begründungspflicht vgl. EuG, Rs. T-308/07, Tegebauer/Parlament vom 14. September 2011, Rn. 24. Zum Meinungsstand bezüglich der Begründungspflicht bei der Entscheidung einer Petition zum Deutschen Bundestag (Art. 17 GG): Ulli Rühl, Der Umfang der Begründungspflicht von Petitionsbescheiden, in: DVBl. 1993, S. 14 - 20, der einer Begründungspflicht nicht Verfassungsrang einräumt, sondern diese allenfalls aus einem Selbstbindungswillen des Parlaments anerkennt. 


\subsection{Die parlamentarische Kontrolle von Handlungen des Petitionsausschusses}

In Verfahren vor dem EuGH zur Nachprüfung von Handlungen des Bürgerbeauftragten argumentierte das EP, es bestehe bereits eine Kontrolle des Bürgerbeauftragten durch das Parlament, daher würde die gerichtliche Überprüfung seiner Tätigkeit eine Verdoppelung der vom Parlament ausgeübten Kontrolle darstellen; außerdem komme die Parlamentskontrolle einer gerichtlichen Kontrolle gleich. ${ }^{65}$ Wendet man dieses Argument (das vom Gericht zurückgewiesen wurde) mutatis mutandis auf die Tätigkeit des Petitionsausschusses an, ergeben sich auch hier einige Vorbehalte, weil die Kontrolle des Ausschusses durch das Plenum sehr limitiert ist. Eine eigenständige Überprüfungsmöglichkeit der Entscheidungen im Sinne einer zweiten Instanz vor dem Plenum gibt es nicht. Zwar hat der Ausschuss das Parlament halbjährlich über die Ergebnisse der Beratungen zu unterrichten ${ }^{66}$, es gibt aber weder für den Petenten noch für das Parlament selbst eine Möglichkeit, ein Petitionsverfahren vor das Plenum zu bringen. Der Ausschuss kann aus eigenem Entschluss einen Initiativbericht ausarbeiten und dem Plenum einen Entschließungsantrag nach Art. 202 Nr. 2 GeschOEP vorlegen. Hierfür steht dem Ausschuss ein weites Ermessen zu. Diese Vorlage eines Entschließungsantrags ist nicht als Revision oder Kontrolle von Handlungen des Petitionsausschusses zu verstehen, sondern nur als eine teilweise Rückdelegation einer Entscheidungsfindung an das Plenum, das somit die Erstentscheidung in der betreffenden Sache trifft. In jedem Fall bleibt es bei der einstufigen Entscheidung des Parlaments, das durch seinen eigenen Ausschuss vollständig repräsentiert wird. Aus der Ausschuss-Einsetzungsbefugnis des Plenums lässt sich also im Ergebnis keine formale Kontrollbefugnis bezüglich getroffener Entscheidungen ableiten. Dagegen bleibt es dem EP unbenommen, sich im Rahmen seiner Beratungen im Plenum mit einer Sachfrage zu befassen, die Gegenstand einer Petition ist oder war. Dabei kann allerdings nicht über die Petition als solche entschieden werden, für die weiterhin der Ausschuss zuständig ist.

\subsection{Die gerichtliche Kontrolle von Handlungen des Petitionsausschusses}

Dem Petenten eröffnet sich eine Kontrollmöglichkeit im Rahmen einer Individualklage gegen Handlungen des Petitionsausschusses vor dem Gericht des EuGH.

\section{Zurechenbarkeit und Rechtswirkungen von Entscheidungen über eine Petition}

Eine mit der Nichtigkeitsklage nach Art. 263 Abs. 4 AEUV angegriffene Handlung muss einem Organ (beziehungsweise einer sonstigen EU-Einrichtung) zurechenbar sein. Wie oben dargelegt, tritt das in Trägerschaft stehende Organ Parlament als Verpflichtungsadressat für seinen Ausschuss ein. ${ }^{67}$ Für die Überprüfbarkeit von Handlungen einer Einrichtung der Union kann die in der Les Verts-Rechtsprechung gegebene Charakterisierung der europäischen Rechtsordnung als Ausgangspunkt dienen: Diese ist „ein umfassendes Rechtsschutz-

65 Rs. C-234/02 P, Bürgerbeauftragter/Lamberts vom 23. März 2004, Rn. $46 f$.

66 Art. 202 Nr. 8 GeschOEP.

67 Vgl. Axel Rolf Schneider, a.a.O. (Fn. 12), S. 116 f.; Eckhard Barth, Bürgerbeauftragter und Petitionsrecht im Prozess der europäischen Verfassungsgebung, Tübingen 2004, S. 141. 
system“, „innerhalb dessen dem Gerichtshof die Überprüfung der Rechtmäßigkeit der Handlungen der Organe übertragen ist" ${ }^{68}$. Handlungen des Parlaments sind also nicht grundsätzlich vom Anwendungsbereich der Nichtigkeitsklage ausgenommen. Zwar hatte das EP ursprünglich nur beratende Funktion und dabei „lediglich“ die Aufgabe der politischen Kontrolle. ${ }^{69}$ Seit dem Urteil zu Les Verts bejaht die Rechtsprechung die in der Rechtssache relevante Frage der Passivlegitimation, aber auch die der Aktivlegitimation ${ }^{70}$ des Parlaments. Entscheidend ist, dass die Handlung Rechtswirkungen erzeugen soll, und zwar unabhängig davon, welche Rechtsnatur oder Form sie im Einzelnen annimmt. ${ }^{71}$ Die gemäß Art. 288 Abs. 5 AEUV nicht verbindlichen Rechtsformen der Empfehlungen und Stellungnahmen sind damit von den anfechtbaren Handlungen regelmäßig ausgenommen (Art. 263 Abs. 4 i.V.m. Abs. 1 AEUV). Gleiches gilt grundsätzlich von den ebenfalls im „Soft Law“ angesiedelten Mitteilungen; allerdings differenziert das Gericht danach, ob eine Mitteilung ihrem Inhalt nach nur Bestimmungen erläutert, oder ob sie „spezifische oder neue Verpflichtungen festlegt" ${ }^{\text {"72 }}$. In letzterem Falle wäre eine dagegen gerichtete Klage (bei Vorliegen der übrigen Voraussetzungen) zulässig.

Die Bedingung, dass eine Handlung ihrer Finalität nach Rechtswirkungen erzeugen soll beziehungsweise tatsächlich Rechtswirkungen erzeugt ${ }^{73}$, ist relativ offen und unbestimmt. Es spielt keine Rolle, ob die Handlung ausdrücklich als „Entscheidung“ bezeichnet wird ${ }^{74}$, sondern es kommt auf deren Wesen ${ }^{75}$ an. Gemäß der Rechtsprechung sind nur solche Maßnahmen nachprüfbar als anfechtbare Handlungen im Sinne des Art. 263 Abs. 4 AEUV, die „verbindliche Rechtswirkungen erzeugen, welche die Interessen des Klägers durch einen Eingriff in seine Rechtsstellung beeinträchtigen "76. Die neuere ständige Rechtsprechung 77

68 EuGH, Rs. 294/83, Les Verts vom 23. April 1986, Rn. 23; EuGH, Rs. 314/85, Foto-Frost vom 22. Oktober 1987, Rn. 16.

69 EuGH, Rs. 294/83, Les Verts vom 23. April 1986, Rn. 24.

70 Vgl. ebenda, Rn. 22 am Ende.

71 EuGH, Rs. C-57/95, Frankreich/Kommission vom 20. März 1997, Rn. 7; EuGH, Rs. 294/83, Les Verts vom 23. April 1986, Rn. 24. Walter Frenz, a.a.O. (Fn. 18), Bd. 5, S. 804, Rn. 2770.

72 EuGH, Rs. T-258/06, Deutschland/Kommission vom 20. Mai 2010, Rn. 28. Zur Einordnung von Soft Law, vgl. Jürgen Schwarze, Soft Law im Recht der Europäischen Union, in: EuR 2011, 3 ff., S. 9; Peter-Christian Müller-Graff, Das Soft Law der europäischen Organisationen, in: EuR 2012, S. 18 ff.; Oliver Mader, Verteidigungsrechte im Europäischen Gemeinschaftsverwaltungsverfahren, Baden-Baden 2006, S. 296. Auch die Mitteilung, eine Beschwerde zu den Akten zu legen, stellt einen das Verfahren abschließenden und daher angreifbaren Beschluss dar: Jürgen Schwarze, in: ders., a.a.O. (Fn. 9), Art. 263 AEUV, Rn. 27; EuGH, Rs. C-39/93 P, SFEI u.a./ Kommission vom 16. Juni 1994, Rn. 28.

73 Die Rechtsprechung ist hier uneinheitlich und lässt bisweilen das finale Element als Erfordernis fortfallen, so dass die tatsächliche Rechtswirkung einer Maßnahme ausreicht: EuGH, Rs. 307/81, Alusuisse Italia/Rat und Kommission vom 6. Oktober 1982, Rn. 8; sowie die unten in Fn. 75 und 77 genannte IBM-Rechtsprechung.

74 Ständige Rechtsprechung, vgl. Rs. C-325/91, Frankreich/Kommission, Rn. 9. Markus Kotzur, a.a.O. (Fn. 12), Art. 263, Rn. 11.

75 EuGH, Rs. C-322/09 P, NDSHT Nya Destination Stockholm Hotell \& Teaterpaket AB/Kommission, Rn. 46.

76 EuGH, Rs. 60/81, IBM/Kommission vom 11. November 1981, Rn. 9.

77 EuGH, Rs. C-362/08 P, Internationaler Hilfsfonds/Kommission vom 26. Januar 2010, Rn. 51; EuGH, Rs. C-131/03 P, Reynolds Tobacco u.a./Kommission vom 12. September 2006, Rn. 54 (mit weiteren Nachweise); EuGH, Rs. C-322/09 P, NDSHT Nya Destination Stockholm Hotell \& Teaterpaket AB/Kommission, Rn. 45. 
stellt auf die Geeignetheit der Organhandlung ab, „verbindliche Rechtswirkungen [zu] erzeugen, die die Interessen des Klägers durch eine qualifizierte Änderung seiner Rechtsstellung beeinträchtigen“. Bei Handlungen des Europäischen Parlaments und des Europäischen Rates wird gewöhnlich auf die nötige Außenwirkung hingewiesen, das heißt die Rechtswirkung müsse Außenstehenden gegenüber eintreten und nicht nur rein internen Charakter haben. ${ }^{78}$ Die genauere Betrachtung der Justiziabilität von Handlungen des Petitionsausschusses bietet einerseits Gelegenheit, die dogmatischen Grenzlinien anhand der oben dargelegten Systematik zu bestätigen; andererseits gibt deren Anwendung Auskunft darüber, welche Reichweite an Rechtswirkungen die Entscheidungen des Ausschusses im Lichte eines modernen Grundrechtsverständnisses erzeugen. Zu diesem Zwecke werden hier die Handlungsoptionen des Ausschusses und die entsprechenden Rechtswirkungen für den Petenten systematisch analysiert.

\section{Szenarien für die Behandlung von Petitionen}

Der Beschluss des Ausschusses, der nach gewöhnlicher Behandlung der Petition ein Petitionsverfahren abschließt, hat den Charakter einer Empfehlung ${ }^{79}$, entfaltet keine Zwangswirkung und auch sonst regelmäßig keine Rechtswirkung. Selbst die Annahme eines Berichts in Kombination mit einem vom EP verabschiedeten Entschließungsantrag, was im Hinblick auf das Spektrum seiner politischen Machtausübung die stärkste Handlungsweise des Petitionsausschusses darstellen dürfte, wird so zu beurteilen sein. Will sich ein Petent gegen die Behandlung seiner Sache durch den Petitionsausschuss wehren, kommen drei denkbare Szenarien für die Klageerhebung des Petenten ${ }^{80}$ gegen Maßnahmen des Ausschusses in Betracht:

(1) Die Petition wird durch den Petitionsausschuss als unzulässig abgewiesen (und dementsprechend nicht behandelt). Hier ist die Möglichkeit einer Nichtigkeitsklage gemäß Art. 263 Abs. 4 AEUV eröffnet. ${ }^{81}$ Der Grund für diesen Rechtsschutz besteht darin, dass dem Petenten die Nachprüfbarkeit der richtigen Einschätzung über das Vorliegen eines Petitionsrechts vorbehalten bleiben soll. Eine unberechtigte Ablehnung kann „das Petitionsrecht der

78 Vgl. Claus Dieter Classen, in: Reiner Schulze / Manfred Zuleeg / Stefan Kadelbach, a.a.O. (Fn. 9), S. 218, Rn. 19; Jürgen Schwarze, a.a.O (Fn. 72), Art. 263 AEUV, Rn. 31. Angreifbar sind Entscheidungen, die ein Verfahren verbindlich abschließen oder sonst eine besondere verbindliche Wirkung erzielen, nicht aber Zwischenmaßnahmen, die die abschließende Entscheidung nur vorbereiten, EuGH, Rs. C-282/95 P, Guérin automobiles vom 18. März 1997, Rn. 34. Der EuGH trennt in Rs. 60/81, IBM/Kommission vom 11. November 1981, Rn. 19 ff. die Rechtswirkung von der "tatsächliche[n] Folge“; die bloße Einleitung eines Verfahrens oder die Mitteilung der Beschwerdepunkte sind danach keine separat angreifbaren Handlungen (ebenda Rn. 21). Zur Justiziabilität investigativer Maßnahmen von OLAF: Jan Inghelram, Judicial Review of Investigative Acts of the European Anti-Fraud Office (OLAF): A Search for a Balance, in: CML Rev. 2012, S. 601 - 627, S. 605 ff.; ders., Legal and Institutional Aspects of the European Anti-Fraud Office (OLAF), Groningen 2011, S. 203 ff.

79 Vgl. Axel Rolf Schneider, a.a.O. (Fn. 12), S. 154.

80 Auf Klagen Dritter soll hier nicht eingegangen werden.

81 Vgl. Walter Frenz, a.a.O (Fn. 18), Bd. 6, S. 80, Rn. 290; Marcel Haag, a.a.O. (Fn. 51), Art. 194, Rn. 18; Johann Schoo / Niklas Görlitz, a.a.O. (Fn. 39), Art. 227 AEUV, Rn. 15; Charlotte Gaitanides, in: Sebastian Heselhaus / Carsten Nowak, a.a.O. (Fn. 9), S. 1326, Rn. 29. 
Bürger, wie es im Vertrag verankert ist, in seinem Wesen beeinträchtigen" 82 , wie das Gericht im Urteil zur Rechtssache Tegebauer feststellte. Die Zurückweisung ist dem Petenten gegenüber zu begründen ${ }^{83}$, wobei eine solche Entscheidung die Gründe, die eine Ablage der Petition ohne weitere Bearbeitung rechtfertigen, „eindeutig erkennen lassen“ 84 muss. Im Erfolgsfall liegt dem Petenten eine durch das Gericht ausgesprochene Nichtigerklärung der die Zulässigkeit der Petition abweisenden Entscheidung des Ausschusses vor.

(2) Wird eine für zulässig befundene Petition durch den Petitionsausschuss einer Prüfung unterzogen, so steht gegen die darauf erfolgende Maßnahme keine Klagemöglichkeit offen, weil dem Ausschuss hinsichtlich der Ausgestaltung seiner Untersuchungen, seiner Methode und auch der Ergebnisse freie Hand gelassen werden soll, seine eigene politische Ermessensentscheidung zu treffen. Da vorliegend davon ausgegangen wird, dass die Entscheidungen des Ausschusses keine Rechtswirkungen entfalten, ist es auch nicht erforderlich, die Reichweite einer solchen politischen Ermessensentscheidung wenigstens durch eine Art Willkürverbot zu beschränken. Diese Konstellation ist einer Nachprüfung durch den Unionsrichter also insgesamt entzogen. Das Gericht räumt dem Parlament in dieser Fallgruppe „insoweit eine vollständige Beurteilungsfreiheit [ein], die politischer Natur ist" ${ }^{\text {" }}$.

(3) Als dritte Variante ist denkbar, dass eine Petition zwar zunächst als zulässig eingeordnet wird, der Ausschuss sie jedoch dann nicht behandelt, das heißt nicht „prüft“ im Sinne des Art. 202 Nr. 1 GeschOEP. Sowohl Wortlaut als auch Verfahrenssystematik der Geschäftsordnung gehen davon aus, dass jede zulässige Petition auch in sachlicher Hinsicht geprüft wird, sei es durch Bericht, durch Stellungnahme oder auf andere Weise. ${ }^{86}$ Teils wurde allerdings vom Petitionsausschuss beziehungsweise vom EP die Auffassung vertreten, eine Ablage oder einfache Abgabe an andere Stellen sei ebenfalls als Prüfung zu werten. Der Begriff der Prüfung einer Petition im Sinne der Geschäftsordnung ist daher von Bedeutung. Er kann Aufschluss über die Kernfrage der Abgrenzung geben, nämlich wo der einklagbare Individualanspruch des Grundrechts auf Petition endet und wo das nicht justiziable „freie politische Ermessen“ des Petitionsausschusses beginnt.

\section{Pflicht zur „Prüfung“ einer Petition versus „freies politisches Ermessen“}

Ein Petent hat zwar keinen Anspruch darauf, dass das von ihm vorgebrachte Begehren erfüllt wird, selbst dann nicht, wenn es begründet ist; aber der Petent hat einen Anspruch darauf, dass sich das Europäische Parlament beziehungsweise dessen Petitionsausschuss mit der Petition befasst. Wie diese Prüfung im Einzelnen auszusehen hat, bleibt dem Ausschuss überlassen. Jedoch muss eine inhaltliche Prüfung stattfinden und eine Stellungnahme erfolgen, wenn die Petition zulässig ist. ${ }^{87}$

82 EUG, Rs. T-308/07, Tegebauer/Parlament vom 14. September 2011, Rn. 21.

83 Art. 201 Nr. 8 GeschOEP.

84 EUG, Rs. T-308/07, Tegebauer/Parlament vom 14. September 2011, Rn. 24.

85 Ebenda, Rn. 21. So auch Johann Schoo / Niklas Görlitz, a.a.O. (Fn. 39), Art. 227 AEUV, Rn. 15 f., ohne allerdings auf die Nichtbehandlung zulässiger Petitionen einzugehen.

86 Vgl. Thomas Giegerich, a.a.O. (Fn. 9), S. 374, Rn. 119.

87 Vgl. Siegfried Magiera, a.a.O. (Fn. 18), Art. 44, Rn. 7; Walter Frenz, a.a.O. (Fn. 18), Bd. 4, Berlin u.a. 2009 , S. 1448, Rn. 4779. 


\section{(a) Bloße Verweisung ist grundsätzlich keine Prüfung}

Eine bloße Verweisung der Petition an eine andere Stelle stellt grundsätzlich keine „Prüfung“ dar. Der Extremfall einer durch Abgabe verweigerten Prüfung wäre die Verweisung an genau diejenige Behörde, gegen deren Verhalten sich eine Petition richtet; eine solche Abgabe würde dem Petenten „Steine statt Brot“ geben. Die bloße Weiterverweisung oder Ablage einer Petition ohne Möglichkeit der Überprüfung würde den Bürger in solchen Fällen seines Petitionsrechtes berauben, in denen er eine zulässige Petition eingereicht hat, aus der dann aber Folgerungen gezogen werden, die nur im Falle einer unzulässigen Petition gerechtfertigt sind, nämlich die Nichtprüfung und Ablage der Petition. Mit anderen Worten: die Ablage oder rechtswidrige Weiterverweisung einer zulässigen Petition ohne Prüfung beinhaltet einen größeren Rechtsbruch als die Nichtprüfung einer fälschlich für „unzulässig“ gehaltenen und als solche abgelegten zulässigen Petition. Denn letztere legt bereits ihrer äußeren Form nach („Unzulässigkeit“) dem Petenten eine Nachprüfungsmöglichkeit in die Hände. Hinsichtlich ihrer Überprüfbarkeit sind beide Fälle gleichzustellen.

Nach dem oben zum institutionellen Verhältnis zwischen Petitionsausschuss und Ombudsmann Gesagten erscheint als einzige Ausnahme zum Verweisungsverbot die erlaubte Weiterleitung einer Petition an den Bürgerbeauftragten, und zwar nach Genehmigung durch den Petenten. Es kann dahinstehen, ob eine solche Weiterleitung als ein Fall der erfolgten „Prüfung" der Petition aufgefasst werden muss oder als eine ungeschriebene gestattete Ausnahme von der Prüfungspflicht. Man kann argumentieren, dass die Verweisung entweder ein Spezialfall der Prüfung oder eine geregelte Ausnahme von der Prüfung ist. Für ersteres spricht, dass sich der Ausschuss zum Zwecke dieser Behandlung unter Umständen zuerst inhaltlich mit der Sache befassen musste, um dann nach Zweckmäßigkeitserwägungen festzustellen, dass der Bürgerbeauftragte die geeignetere Einrichtung darstellt und die Genehmigung durch den Petenten einholt. Für die zweite Auffassung spricht, dass die Möglichkeit der Weiterleitung ausdrücklich in Art. 201 Nr. 12 der Geschäftsordnung genannt ist, also auf derselben Ebene, auf der auch die Prüfungspflicht geregelt ist. Dafür würde auch sprechen, dass in vielen Fällen (aber eben nicht immer) eine Entscheidung zur Zweckmäßigkeit der Behandlung durch die eine oder andere Einrichtung ohne eine inhaltliche Analyse des Vorbringens erfolgen kann. So würde also keine „Prüfung" vorgenommen werden müssen, um über eine Verweisung entscheiden zu können. Gegen diese Ansicht (und damit eher für die erstgenannte Auffassung) würde sprechen, dass die Pflicht zur Prüfung einer Petition auch und gerade aus höherrangigem (Verfassungs-)Recht abzuleiten ist, in dem die Weiterleitungsmöglichkeit an den Bürgerbeauftragten gerade nicht vorgesehen ist. Eine „geregelte Ausnahme von der Prüfungspflicht" ist auf der Ebene des Verfassungsrechts nicht ersichtlich.

\section{(b) Versagung des Grundrechts als qualifizierte Änderung der Rechtsstellung des Petenten}

Hier wird vertreten, dass die von der Rechtsprechung entwickelte Methode der Ermittlung, ob die Maßnahme eine nach Art. 263 Abs. 4 AEUV anfechtbare Handlung darstellt, auch im Zusammenhang mit Petitionsentscheidungen anwendbar ist. Es ist also danach zu fragen, ob die angegriffene Handlung des Petitionsausschusses dazu bestimmt war, Rechtswirkungen zu erzeugen, oder diese jedenfalls de facto erzeugt. Angesichts der Breite der Tätigkeit, der Handlungs- und Entscheidungsmöglichkeiten des Ausschusses ist zwar nicht a priori auszuschließen, dass eine nach Prüfung der Petition erfolgende Entscheidung im Einzelfall - obwohl sie als Bericht, Stellungnahme oder Empfehlung in Erscheinung tritt - Rechtswirkun- 
gen erzeugt. Im Regelfall jedoch wird es sich um eine politische Stellungnahme oder kritische Meinungsäußerung ohne Rechtswirkung handeln mit der Folge, dass eine gerichtliche Überprüfung ausscheidet.

Dass eine gerichtliche Überprüfbarkeit gegeben sein muss, wenn - wie oben beschrieben - eine Petition zwar zunächst als zulässig eingeordnet wird, sie aber dann nicht geprüft wird, ergibt sich aus der Überlegung, dass der Petitionsausschuss zur wirksamen Behandlung 88 einer Petition verpflichtet ist. Nur eine solche kann in diesem Sinne eine Prüfung nach Art. 202 Nr. 1 der GeschOEP sein. Mit der Behandlung und Bescheidung einer Petition erfüllt der Ausschuss sowohl seine eigene Pflicht als auch das Recht des Petenten aufgrund der Leistungs- und Schutzfunktion des Grundrechts. Ohne eine solche Pflicht beziehungsweise ein solches Recht wäre die Regelung der Petition „praktisch funktionslos" ${ }^{89}$, sie wäre reduziert auf einen Spezialfall des Rechts auf freie Meinungsäußerung. ${ }^{90}$ Diese Pflicht ergibt sich außer aus den genannten Grundrechtsnormen auch aus der selbstbindenden Vorschrift des Art. 202 der Geschäftsordnung des Parlaments, wonach das EP verpflichtet ist, die gemäß Art. 201 Abs. 7 GeschOEP für zulässig befundenen Petitionen zu prüfen und den Petenten über die Begründung für den vom Ausschuss gefassten Beschluss zu informieren. Der Petitionsausschuss legt durch seine Entscheidungen den Standpunkt ${ }^{91}$ des Parlaments im Hinblick auf die Behandlung einer Petition fest und verändert dadurch die Rechtsstellung des Petenten. Eine Verweigerung der inhaltlichen Behandlung der Petition hindert den Petenten an der wirksamen Wahrnehmung seines Petitionsrechts. Sie ist geeignet, die Interessen des Klägers durch eine qualifizierte Änderung seiner Rechtsstellung zu berühren, weil es sich um die Verletzung der Substanz eines Grundrechts handelt. ${ }^{92}$ Hinsichtlich des „Ob“ der Prüfung einer zulässigen Petition hat der Ausschuss also kein Ermessen, hinsichtlich des "Wie“ kommt ihm dagegen ein politisches Ermessen zu, das aufgrund der Natur des Instruments außergewöhnlich weit ist. ${ }^{93}$

88 Siegfried Magiera, a.a.O. (Fn. 18), Art. 44, Rn. 7; Asteris Pliakos, in : CDE (Cahiers de droit européen) 1993, S. 348: „(...) le Parlement ayant l'obligation de traiter efficacement une pétition (...). Aussi tout traitement inefficace d'une pétition doit-il équivaloir à la violation de cette obligation. "Zur Pflicht der sachlichen Prüfung im deutschen Recht (Art. 17 GG): BVerfGE 2, S. 225 (S. 229).

89 Marcel Haag, a.a.O. (Fn. 51), Art. 194, Rn. 8. Ebenso Walter Frenz, a.a.O. (Fn. 18), Bd. 4, Berlin u.a. 2009, S. 1448, Rn. 4779; Hans Dieter Jarass, EU-Grundrechte, München 2005, \$ 5, Rn. 10, 11.

90 Vgl. Axel Rolf Schneider, a.a.O. (Fn. 12), S. 103.

91 Zum Begriff der Festlegung des Standpunktes vgl. Rs. C-322/09 P, NDSHT Nya Destination Stockholm Hotell \& Teaterpaket AB/Kommission, Rn. 48.

$92 \mathrm{Zu}$ einem ähnlichen Ergebnis hinsichtlich der Rechtswirkungen im Parallelfall der Verweigerung des Grundrechts auf Anhörung gelangt Jan Inghelram, a.a.O. (Fn. 78), S. 611, unter Verweis auf EuGH verbunden Rs. C-402/05 P und C-415/05 P, Kadi und Al Barakaat International Foundation/Rat und Kommission vom 3. September 2008, Rn. 285, 304; ders., Fundamental Rights, the European Anti-Fraud Office (OLAF) and a European Public Prosecutor's Office (EPPO), in: KritV 2012, S. $67-81$, S. 77: „In any case, depriving a person of the exercise, or the benefit, of a fundamental right amounts, in fact, to taking away, entirely or partly, that right from its beneficiary", unter Verweis auf EuGH, Rs. T-16/91, Rendo u.a./Kommission vom 18. November 1992, Rn. 54f. (Verfahrensrechtsverstoß). Zu den Rechtsfolgen der Versagung von Verfahrensgrundrechten: Oliver Mader, a.a.O. (Fn. 72), S. 365 ff, S. 404 ff.

93 Auch hier gibt es eine Parallele zum Bürgerbeauftragten, dem ein weites Ermessen zukommt, und der ebenso wenig wie der Petitionsausschuss verpflichtet ist, ein bestimmtes Ergebnis zu erzielen. 
Zusammenfassend kann gesagt werden, dass der Petitionsausschuss zwar regelmäßig keine Maßnahmen mit Rechtswirkung treffen wird, wenn er seine Untersuchungen vornimmt sowie Berichte und Stellungsnahmen ausarbeitet, selbst dann, wenn diese über einen Entschließungsantrag vom Plenum angenommen werden. Dagegen stellt die Ablehnung der inhaltlichen Behandlung einer zulässigen Petition eine Maßnahme dar, die gegen die wirksame Wahrnehmung des Petitionsrechts gerichtet ist und daher einer gerichtlichen Anfechtung zugänglich sein muss.

\section{Richtige Klageart}

Richtige Rechtsschutzform wird regelmäßig die Nichtigkeitsklage nach Art. 263 Abs. 4 AEUV sein, die gegen die an den Petenten versandte Mitteilung über den Abschluss des Petitionsverfahrens gerichtet ist. Dies gilt selbst bei nur geringfügigem Tätigwerden nach außen, etwa einer Mitteilung über den Abschluss des Verfahrens ohne Behandlung in der Sache. Eine Untätigkeitsklage ${ }^{94}$ nach Art. 265 AEUV kommt nur bei völliger Untätigkeit des Parlaments beziehungsweise seines Petitionsausschusses in Frage. Die damit unterlassene Petitionsbescheidung ist ein verbindlicher Akt, der im hypothetischen Falle seines Bestehens mit der Nichtigkeitsklage angegriffen werden könnte. ${ }^{95}$

\section{Würdigung der jüngeren Rechtsprechung und Ergebnis}

Das Petitionsrecht beim Europäischen Parlament ist ein Recht auf Verfahren und ein Recht im Verfahren. Sein Schutzbereich ist in persönlicher Hinsicht weit gefasst und beinhaltet sachlich die Entgegennahme der Petition, ihre inhaltliche Prüfung bei Zulässigkeit und eine begründete Entscheidung, die dem Petenten mitzuteilen ist. Dieser hat keinen Anspruch auf ein bestimmtes Ergebnis oder eine spezifische Handlungsweise des durch den Petitionsausschuss repräsentierten Parlaments. Ist die Petition zulässig, hat der Petent aber einen Anspruch auf Behandlung und inhaltliche Stellungnahme.

Das Urteil in der Rechtssache Tegebauer und die Konstellation des im Frühjahr 2013 zu erwartenden Urteils in der Rechtssache Schönberger liegen auf der Linie eines erhöhten Grundrechtsschutzes. Das Gericht hatte in ersterem Fall lediglich die Frage zu beantworten, ob die Entscheidung des Ausschusses, eine Petition für unzulässig zu erklären, der Nachprüfung durch den Unionsrichter unterliegt. Es hat diese im Sinne der Wirksamkeit des Petitionsrechts bejaht: Die Petition ohne weitere Bearbeitung abzulegen habe Auswirkungen für den Inhaber dieses Rechts, da seine Petition für unzulässig erklärt und aufgrund dessen inhaltlich nicht geprüft worden ist.

In der Rechtssache Schönberger erhielt der Petent durch die Vorsitzende des Ausschusses die Benachrichtigung, dass sich der Ausschuss mit der (zulässigen) Petition nicht befassen

Auch sein Handeln ist nicht von einer Nachprüfbarkeit ausgeschlossen. Vgl. EuGH im Urteil zu Rs. C-234/02 P, Bürgerbeauftragter/Lamberts vom 23. März 2004, Rn. $51 f$ (dort über eine Amtshaftungsklage). Zu den (engen) Grenzen der Nachprüfbarkeit: Oliver Mader, a.a.O. (Fn. 72), S. 361.

94 Vgl. Johann Schoo, a.a.O. (Fn. 39), Art. 227 AEUV, Rn. 14; Walter Frenz, a.a.O. (Fn. 18), Bd. 4, S. 1446, Rn. 4773.

95 Vgl. Charlotte Gaitanides, a.a.O. (Fn. 9), S. 1328, Rn. 38. 
könne. Die Petition wurde an die Verwaltungsstelle weiterverwiesen, deren frühere Handlungsweise der Petent kritisierte und ihm wurde mitgeteilt, dass das Petitionsverfahren abgeschlossen sei. Damit erhält das Gericht die Möglichkeit, über die in Tegebauer konstatierte Überprüfbarkeit der Unzulässigkeitsentscheidung noch einen Schritt hinaus zu gehen und auch die Nichtbehandlung einer zulässigen Petition dem Fall gleichzustellen, in dem der Ausschuss fälschlicherweise die Unzulässigkeit einer Petition angenommen hatte. Beide Aspekte zusammen genommen ergeben einen vollständigen Schutz der Wirksamkeit des Petitionsrechts in seiner unionsrechtlichen Justiziabilität.

Das Ergebnis fügt sich nahtlos ein in die von der Rechtsprechung entwickelte Systematik zur Qualifikation von Organverhalten, das mit der Nichtigkeitsklage gemäß Art. 263 Abs. 4 AEUV angegriffen werden kann. Danach sind nur Maßnahmen justiziabel, die „verbindliche Rechtswirkungen erzeugen, die die Interessen des Klägers durch eine qualifizierte Änderung seiner Rechtsstellung beeinträchtigen "96. Das freie politische Ermessen bei inhaltlichen Entscheidungen des Petitionsausschusses anlässlich der (tatsächlichen) Behandlung von Petitionen entfaltet keine verbindlichen Rechtswirkungen und unterliegt keiner Nachprüfung durch den Unionsrichter. Die Entscheidung dagegen, das Grundrecht auf Petition zu versagen, erzeugt verbindliche Rechtswirkungen gegenüber dem Petenten und muss zur Gewähr der Wirksamkeit dieses Rechts nachprüfbar bleiben. Dies gilt gleichermaßen für eine fälschlich unzulässige wie für eine zulässige, aber dennoch unbehandelt gebliebene Petition.

96 EuGH, Rs. C-131/03 P, Reynolds Tobacco u.a./Kommission vom 12. September 2006, Rn. 54.

\title{
Klingel oder Gong? Akustische Signale in der parlamentarischen Praxis
}

\author{
Kai Zähle
}

Akustische Signale gehören zu den parlamentarischen Besonderheiten. Im Laufe eines Sitzungstages können etwa Klingel oder Gong parlamentarische Anlässe ankündigen, und bei hitzigen Debatten kann Glockengeläut erklingen, um die angemessene Ruhe wieder herzustellen. Mancher Besucher wird durch dieses Klangerlebnis verunsichert, weil er zwar die Glocken hört, aber nicht weiß, wo sie hängen. Das geübte Ohr empfindet akustische Signale hingegen als Bereicherung und weiß, was die Glocke geschlagen hat, wenn solche Signale erklingen. ${ }^{1}$ Wie schon der Bundestagsabgeordnete Wolfgang Bosbach betonte, horchen die Parlamentarier auf und reagieren rasch, wenn Töne dieser Art erschallen. ${ }^{2}$ In der parlamentarischen Praxis haben sich unterschiedliche Signalerzeugungen etabliert, um die Aufmerksamkeit der Abgeordneten zu erreichen. Ziel dieser Miszelle ist es, Einblick in das System

1 Siehe zu diesen Redensarten Duden, Das große Wörterbuch der deutschen Sprache, Bd. 4, Mannheim 1999, Stichwort Glocke, S. 1541; Lutz Röhrich, Das große Lexikon der sprichwörtlichen Redensarten, Bd. 1, Freiburg 1991, Stichwort Glocke, S. 555.

2 Vgl. Wolfgang Bosbach, Volksvertreter, hört die Signale!, http://wobo.de/deutscher_bundestag/ artikel_bundestag/die-signale-im-deutschen-bundestag-1.pdf (Abruf am 27. Juni 2011). 